Maria Helena Palucci Marziale

Everaldo Jose da Silva ${ }^{2}$

Vanderley José Haas ${ }^{3}$

Maria Lúcia do C. C. Robazzi ${ }^{4}$

${ }^{1}$ Enfermeira. Professora Titular da Escola de Enfermagem da Universidade de São Paulo, Ribeirão Preto-SP, Brasil.

${ }^{2}$ Enfermeiro do Trabalho do Hospital Universitário de Brasília-DF, Brasil.

${ }^{3}$ Físico. Doutor em Ciências. Professor Prodoc da Escola de Enfermagem da Universidade de São Paulo, Ribeirão Preto-SP, Brasil.

${ }^{4}$ Enfermeira. Professora Titular da Escola de Enfermagem da Universidade de São Paulo, Ribeirão Preto-SP, Brasil.

\section{Acidentes com material biológico em hospital da Rede de Prevenção de Acidentes do Trabalho - REPAT}

Accidents involving biological material in a hospital from the Network on Work Accident Prevention - REPAT

\begin{abstract}
Resumo
Estudo transversal com objetivo de descrever os acidentes do trabalho com exposição a material biológico ocorridos no Hospital Universitário de Brasília e analisar as ações preventivas utilizadas na instituição. Para a coleta dos dados, foi utilizado o formulário eletrônico da Rede de Prevenção de Acidente do Trabalho (REPAT). Os dados foram coletados nos anos de 2003 e 2004 e analisados estatisticamente por meio de cálculos de freqüência. Dentre 2.000 a 2.011 trabalhadores atuantes no período, foi constado o registro de 107 acidentes. O maior número de acidentes ocorreu entre mulheres, no período da manhã, com lesão perfurante nas mãos com agulhas e cateteres, nas unidades de Clínica Médica e Pronto Atendimento. As ações preventivas adotadas são: treinamento, visitas aos locais de trabalho e orientações individuais, as quais, diante das características dos acidentes encontrados, precisam ser revistas e ampliadas.
\end{abstract}

Palavras-chaves: acidentes do trabalho, saúde ocupacional, saúde do trabalhador, risco biológico.

\begin{abstract}
This transversal study is aimed at diagnosing work accidents involving exposure to biological material occurred at the Brasília University Hospital, as well as at analyzing institutional preventive actions. The REPAT - Work Accident Prevention Network - electronic form was used for data collection. Data were collected during 2003 and 2004 and the statistical analysis was performed through computation of frequencies. Among the 2.000 to 2.011 workers in action at that time, 107 accidents were evidenced. The highest amount of them occurred in the morning, among women, and involved perforating lesion in hands, caused by needles and catheters in the Medical Clinic and emergency units. Due to the characteristics of these accidents, the preventive actions, which included training, visits to work places and individual orientation, need to be revised and expanded.
\end{abstract}

Keywords: work accidents, occupational health, workers' health, biological risk. 


\section{Introdução}

A exposição ocupacional a material biológico representa um risco para os trabalhadores das instituições de saúde devido à possibilidade de transmissão de patógenos, como o vírus da hepatite B (HBV) e da Síndrome da Imunodeficiência Adquirida (HIV). As conseqüências dessa exposição podem afetar diretamente os trabalhadores, atingindo-os em seus aspectos físico e psicológico e ainda pode repercutir nas relações familiares e sociais (CDC, 2001; MARZIALE, 2003).

Acidentes ocasionados por picadas de agulhas são responsáveis por $80 \%$ a $90 \%$ das transmissões de doenças infecciosas entre trabalhadores de saúde e o risco de transmissão de infecção de uma agulha contaminada é de um em três para a Hepatite $\mathrm{B}$, um em trinta para hepatite $\mathrm{C}$ e um em trezentos para o HIV (GODFRE, 2001). Nos Estados Unidos, estima-se que aproximadamente 8 milhões de trabalhadores de saúde são anualmente vítimas de acidentes com material perfurocortante e os estudantes de Medicina, Odontologia e Enfermagem também conformam um grupo muito atingido por esse tipo de injúria. Dos 16.922 acidentes documentados nos Estados Unidos no período de 1995 a 2001 , cerca de $44 \%$ vitimou enfermeiras, 28\% médicos, 15\% técnicos de laboratório, $4 \%$ estudantes e $3 \%$ pessoal de limpeza (CDC, 2005).

No Brasil, embora os Acidentes do Trabalho (AT) com exposição a material biológico sejam freqüentes, não existe ainda um real diagnóstico do número de trabalhadores acidentados e das conseqüências causadas por essas injúrias, o que tem dificultado o planejamento e a adoção de medidas preventivas.

Neste contexto, foi criada a Rede de Prevenção de Acidentes do Trabalho com Material Biológico em hospitais brasileiros (REPAT). Trata-se de uma rede colaborativa de pesquisas e intercâmbio de informações envolvendo 14 hospitais de várias regiões do país, integrando pesquisadores e especialistas em saúde do trabalhador, que tem como meta o controle e a prevenção de acidentes de trabalho com exposição a material biológico (REPAT, 2007) ${ }^{5}$.
O meio eletrônico foi selecionado pela valiosa contribuição que oferece à pesquisa científica, sendo que a Internet tem sido considerada uma ferramenta vital para colaboração na comunidade de pesquisa (PERINE, 2000). Os pesquisadores podem explorar problemas complexos e interdisciplinares, ter acesso a bancos de dados e consultar colegas de todo o mundo. A rede permite ainda ao pesquisador pensar globalmente e gerar, com maior eficiência e rapidez, produtos de valor para a comunidade, acelerando a difusão do conhecimento científico e o fortalecimento dos paradigmas dominantes (MEDEIROS, 2003).

Assim, através da via eletrônica, buscamos estudar os acidentes do trabalho com exposição a material biológico em hospitais de diferentes estados com a finalidade de: identificar sua ocorrência e possíveis casos de sub-notificação com o objetivo de elaborar medidas preventivas; identificar a ocorrência destes acidentes; e estimular o registro e o controle dos mesmos nos hospitais.

A existência provável de subnotificação de acidentes de trabalho entre profissionais da saúde tem sido apontada na literatura (NAPOLEÃO, ROBAZZI \& MARZIALE, 2000; MARZIALE, NISHIMURA \& FERREIRA, 2004) devido à falta de conscientização do risco por parte dos trabalhadores e gestores de hospitais, ao medo de desemprego ou perda de emprego pelo trabalhador, à culpabilidade que sente em relação a esse evento, à falta de organização adequada das ações do serviço de atendimento ao trabalhador, às dificuldades do sistema de informação e, ainda, à descrença da importância do acidente do trabalho desta natureza.

Nesse sentido, questiona-se nesta pesquisa:

- Quais as dificuldades que levam os profissionais/alunos da área de saúde a não registrarem os acidentes envolvendo materiais biológicos durante suas atividades em um dos hospitais da REPAT?

\section{Objetivo geral}

- Descrever a ocorrência de acidentes do trabalho com exposição a material biológico ocorridos no hospital universitário de Brasília e as estratégias adotadas para a sua prevenção. 


\section{Objetivos específicos}

- Descrever as características do hospital e da organização do serviço de segurança e medicina do trabalho em relação às condutas de registro e prevenção dos acidentes do trabalho com exposição a material biológico;

\section{Material e método}

Trata-se de um estudo de campo de caráter retrospectivo com desenho transversal desenvolvido em um hospital universitário da cidade de Brasília (HUB).

Os casos foram constituídos por trabalhadores do HUB que sofreram acidentes do trabalho envolvendo exposição a material biológico ocorrido no período de 2003 a 2004 e registrados, pelo enfermeiro do trabalho do hospital, diretamente no formulário REPAT on-line usando o micro computador de sala do Serviço de Saúde Ocupacional.

O formulário REPAT foi construído com base no instrumento proposto pela Occupational Safety and Health Administration (OSHA, 2001), no protocolo de coleta de dados do EPINET (JAGGER \& PERRY, 2001). Este formulário contém dados de identificação do trabalhador aciden-

\section{Resultados e discussão}

\section{Caracterização do hospital}

O Hospital Universitário de Brasília é um hospital geral que atende diversas especialidades. Possui 370 leitos e 2.011 funcionários no seu quadro ${ }^{6}$ distribuídos da seguinte forma: 810 pertencentes ao quadro permanente da Fundação Universidade de Brasília (FUB), 391 cedidos pelo Ministério da Saúde para a prestação de serviço no hospital e 800 prestadores de serviços com contratos de trabalho temporários renovados a cada semestre. Somados a estes, embora não se tenham números exatos, o HUB concentra, ainda, servidores cedidos pela Secretaria de Estado de Saúde do Governo do Distrito Federal e por outras instituições. Do total deste contingente, 598 trabalhadores estão diretamente subordinados à Divisão de Enfermagem (enfermeiros, auxiliares de enfermagem, auxiliares operacionais de serviços diversos (AOSD) e secretários).
- Identificar o número de acidentes do trabalho com exposição a material biológico;

- Identificar os locais de trabalho e as categorias profissionais com maior coeficiente de acidentes do trabalho.

tado, do local/setor de trabalho, descrição da atividade realizada e do acidente (objeto causador, motivos/causas do acidente), medidas adotadas quando da ocorrência do acidente (condutas e tratamento), situação vacinal do trabalhador.

O estudo é parte integrante do projeto REPAT, que foi aprovado pelo Comitê de Ética em Pesquisa da Escola de Enfermagem de Ribeirão Preto da Universidade de São Paulo, obedecendo às recomendações da Resolução 196 (BRASIL, 1997).

Para descrever as características do hospital e da organização do serviço de segurança e medicina do trabalho em relação às condutas de registro e prevenção dos acidentes do trabalho com exposição a material biológico, foi realizada uma entrevista semi-estruturada com o enfermeiro do trabalho do hospital.

O hospital atende pacientes do Distrito Federal e de cidades dos Estados de Goiás e Minas Gerais, assim como índios de várias tribos do Brasil, que são encaminhados pela Fundação Nacional de Saúde (FUNASA), e pacientes de convênios particulares.

\section{Serviço especializado de segurança e me-} dicina do trabalho (SESMT)

O serviço foi iniciado em 1992 com a participação de apenas um técnico de segurança do trabalho e um enfermeiro do trabalho, mas, com o passar do tempo, o quadro foi se completando, atendendo à Norma Regulamentadora 4 (BRASIL, 2005), que define os profissionais que devem atuar no SESMT no território nacional. Atualmente, o serviço é composto por três médicos do trabalho, dois enfermeiros do trabalho, uma enfermeira especializada em Saúde Pública, um engenheiro e cinco técnicos em Segurança do Trabalho. Esses

\footnotetext{
${ }^{6}$ Dados da Diretoria de Recursos Humanos do Hospital Universitário de Brasília de 2004
} 
profissionais são subordinados diretamente à Secretaria de Recursos Humanos, que é responsável pelo serviço de segurança do trabalho do hospital.

Dentre as várias atribuições do enfermeiro do trabalho, estão o acompanhamento do AT e o preenchimento da Comunicação de Acidente de Trabalho (CAT) para os profissionais com contrato de trabalho regulamentado pela Consolidação das Leis do Trabalho. Para os servidores com contrato de trabalho regidos pelo Estatuto dos Funcionários Públicos, não cobertos pelo seguro acidente do trabalho do Instituto Nacional do Seguro Social (INSS), os registros são feitos na Comunicação Interna de Acidentes de Trabalho (CIAT), cujo formulário foi recentemente reformulado e ampliado (BOSI, 2002).

A rotina estabelecida para registro e acompanhamento em casos de AT no hospital é descrita a seguir.

$1^{\circ}$. O trabalhador acidentado é avaliado por um médico plantonista, no Setor de Pronto Atendimento, onde lhe são solicitados os exames sorológicos, bem como do paciente-fonte (se for conhecido e desde que este autorize a coleta de sangue), e são prescritos, se necessário, medicamentos anti-retrovirais (Zidovudina, Lamivudina, AZT, 3TC, Indinavir). Geralmente os medicamentos são prescritos por quatro dias, tempo necessário para que os resultados dos exames laboratoriais sejam liberados. No serviço, há condutas diferentes adotadas pelos médicos quanto à duração do tratamento com anti-retrovirais: alguns médicos interrompem o tratamento se os resultados da sorologia para HIV forem negativos; outros, mesmo diante deste resultado, continuam os tratamentos por 30 dias;

$2^{\circ}$. O acidentado deve dirigir-se à farmácia para retirar as medicações e iniciar o tratamento em até duas horas após a exposição ocupacional;

$3^{\circ}$. O trabalhador acidentado comparece ao laboratório para a coleta do sangue e, a seguir, o laboratório se responsabiliza em coletar os exames do sujeito-fonte;

$4^{\circ}$. O último passo é comparecer ao SESMT para efetuar o registro do acidente. Neste momento, o enfermeiro do trabalho orienta o trabalhador sobre a necessidade do uso de Equipamentos de Proteção Individual (EPI) e a necessidade de continuidade dos exames subseqüentes por meio de retornos agendados no período de três e seis meses após o acidente.
Considerando-se que existem recomendações sobre a adequada terapia antiretroviral após acidentes com material biológico (SÃO PAULO, 2002), sugere-se que a instituição estudada deva seguir essas recomendações com a finalidade de uniformizar as suas condutas.

\section{Diagnóstico dos acidentes do trabalho} com exposição a material biológico

Em 2003, estavam alocados no hospital 2.000 trabalhadores e, em 2004, o estafe aumentou para 2.011. Investigando os acidentes registrados no biênio, foi constatada a ocorrência de 107 AT com exposição a material biológico: 54 ocorridos em 2003 e 53, em 2004. Observou-se que $83,3 \%$ dos trabalhadores acidentados eram do sexo feminino e $16,7 \%$ eram do masculino e que $79,21 \%$ dos AT ocorridos em 2004 foram entre mulheres e 20,85\%, entre homens. A predominância de trabalhadores do sexo feminino em hospitais é confirmada na literatura principalmente devido ao grande contingente de mulheres na equipe de enfermagem, população esta de trabalhadores exposta à ocorrência de AT com material biológico (MARZIALE, NISHIMURA \& FERREIRA, 2004; NISHIDE, BENATTI \& ALEXANDRE, 2004).

A Tabela 1 mostra o dia da semana em que os acidentes ocorreram e a Tabela 2 qual o turno de ocorrência dos AT.

Os resultados revelaram que os acidentes ocorreram predominantemente às segundas-feiras $(27,7 \%$ em 2003 e 24,6\% em 2004) e no período da manhã (63\% em 2003 e 54,8\% em 2004). Constatou-se queda acentuada da ocorrência dos AT aos sábados e domingos bem como no período da noite, fato que pode estar relacionado à redução de atividades e do número de trabalhadores expostos ao risco, uma vez que as equipes de trabalho são reduzidas nos finais de semana e no período noturno.

No período da manhã, nos hospitais, geralmente são efetuadas atividades nas quais há manuseio de material perfurocortante, entre os quais cateteres intravenosos, agulhas, lâminas de bisturis entre outros. Considera-se que, devido à rotina adotada, o trabalhador, no período da manhã, está mais exposto a riscos acidentais. Os dados obtidos neste estudo coincidem com os achados de uma pesquisa realizada em hospitais públicos de Rio Branco-Brasil (PEREIRA, 2004). A Tabela 3 mostra os dados relativos ao objeto causador dos acidentes registrados. 
Tabela 1 Distribuição dos acidentes do trabalho com exposição a material biológico no Hospital Universitário integrante do REPAT, no biênio 2003-2004, segundo os dias da semana. Brasília-Brasil

\begin{tabular}{lcccc}
\hline \multicolumn{1}{c}{ Ano } & \multicolumn{2}{c}{2003} & \multicolumn{2}{c}{2004} \\
\multicolumn{1}{c}{ Dia da semana } & $n$ & $\%$ & $n$ & $\%$ \\
\hline Domingo & 3 & 5,6 & 4 & 7,5 \\
Segunda-feira & 15 & 27,7 & 13 & 24,6 \\
Terça-feira & 9 & 16,7 & 8 & 15,1 \\
Quarta-feira & 10 & 18,5 & 9 & 17 \\
Quinta-feira & 6 & 11,1 & 6 & 11,3 \\
Sexta-feira & 9 & 16,7 & 9 & 17 \\
Sábado & 2 & 3,7 & 4 & 7,5 \\
Total & 54 & 100 & 53 & 100 \\
\hline
\end{tabular}

Fonte: Ambulatório de Saúde do Trabalhador, Hospital Universitário de Brasília.

Tabela 2 Distribuição dos acidentes com risco de exposição a material biológico no Hospital Universitário integrante do REPAT, no biênio 2003-2004, segundo o turno da ocorrência. Brasília-Brasil

\begin{tabular}{ccccc}
\hline Ano & \multicolumn{2}{c}{2003} & \multicolumn{2}{c}{2004} \\
Turnos & $n$ & $\%$ & $n$ & $\%$ \\
\hline Manhã & 34 & 63 & 29 & 54,8 \\
Tarde & 13 & 24 & 20 & 37,7 \\
Noite & 7 & 12,9 & 4 & 7,5 \\
Total & 54 & 100 & 53 & 100 \\
\hline
\end{tabular}

Fonte: Ambulatório de Saúde do Trabalhador, Hospital Universitário de Brasília.

Tabela 3 Distribuição dos acidentes com risco de exposição a material biológico no Hospital Universitário integrante do REPAT, no biênio 2003-2004, segundo o objeto causador. Brasília-Brasil

\begin{tabular}{lcccc}
\hline \multicolumn{1}{c}{ Ano } & \multicolumn{2}{c}{2003} & \multicolumn{2}{c}{2004} \\
\multicolumn{1}{c}{ Objeto causador } & $n$ & $\%$ & $n$ & $\%$ \\
\hline Agulha e cateter endovenoso & 42 & 77,7 & 47 & 88,8 \\
Sangue/secreção em mucosa/pele & 6 & 11,2 & 2 & 3,8 \\
Lima usada na Odontologia & 1 & 1,8 & - & - \\
Lâmina vidro/bisturi & 3 & 5,6 & 1 & 1,8 \\
Tubo de vidro & 2 & 3,7 & 2 & 3,8 \\
Porta enferrujada & - & - & 1 & 1,8 \\
Total & 54 & 100 & 53 & 100 \\
\hline
\end{tabular}

Fonte: Ambulatório de Saúde do Trabalhador, Hospital Universitário de Brasília.

Observa-se que, em 2003, agulhas e cateteres endovenosos foram responsáveis por $77 \%$ das lesões com exposição do acidentado a material biológico, sendo considerados potenciais veículos transmissores de infecções. Em 2004, a freqüência de AT com agulhas e cateteres aumentou para 88,8\%. Em estudo realizado no mesmo hospital sobre a ocorrência acidentária no período de julho de 2002 a julho de 2003, identificou-se que $62,8 \%$ dos acidentes ocorridos envolviam objetos perfurocortante (GOMES, 2005). A distribuição dos acidentes segundo parte do corpo atingida encontra-se na Tabela 4.

Pela própria natureza do trabalho, as mãos e os quirodáctilos foram as partes do corpo mais atingidas, com freqüências 
semelhantes nos dois períodos estudados. Constatou-se que os trabalhadores estão sofrendo injúrias também nos pés e nas pernas e essas estão ocorrendo em função do descarte inadequado de material perfurocortante, resultante do posicionamento não adequado dos recipientes de descarte, que devem estar em bancadas em altura de fácil acesso para seus usuários.

Na Figura 1, apresentam-se os resultados relativos ao mês de ocorrência dos AT por meio da análise temporal.

Observa-se, nos resultados apresentados na Figura 1, que, embora não exista tendência ascendente ou descendente para incidência acumulada de AT, durante
2003, houve maior ocorrência de acidentes nos meses de março e outubro e, no ano de 2004, nos meses de janeiro e outubro.

No período estudado, os serviços com maior ocorrência das injúrias foram os setores de clínica médica e pronto atendimento. Esses locais são considerados os setores de maior concentração de pacientes e trabalhadores do hospital estudado. No primeiro local, onde há 66 leitos ativados, estão expostos 102 trabalhadores, enquanto que, no segundo, com 31 leitos, há 89 trabalhadores expostos, sem computar os alunos, cuja rotatividade é grande. $\mathrm{Na}$ Tabela 5, são apresentados os AT segundo os setores do hospital.

Tabela 4 Distribuição dos acidentes com risco de exposição a material biológico no Hospital Universitário integrante do REPAT, no biênio 2003-2004, segundo partes do corpo atingidas. Brasília-Brasil

\begin{tabular}{lcccccc}
\hline \multirow{2}{*}{ Parte do corpo atingida } & \multicolumn{2}{c}{2003} & \multicolumn{2}{c}{2004} & \multicolumn{2}{c}{ Total } \\
& $n$ & $\%$ & $n$ & $\%$ & $n$ & $\%$ \\
\hline Mãos e quirodáctilos & 46 & 85,4 & 47 & 88,8 & 93 & 86,9 \\
Olho(s) & 4 & 7,4 & 2 & 3,8 & 6 & 5,7 \\
Lábios & 1 & 1,8 & - & - & 1 & 0,9 \\
Antebraço & 1 & 1,8 & 2 & 3,8 & 3 & 2,9 \\
Pé & 1 & 1,8 & 1 & 1,8 & 2 & 1,8 \\
Perna & 1 & 1,8 & 1 & 1,8 & 2 & 1,8 \\
Total & 54 & 100 & 53 & 100 & 107 & 100 \\
\hline
\end{tabular}

Fonte: Ambulatório de Saúde do Trabalhador, Hospital Universitário de Brasília.

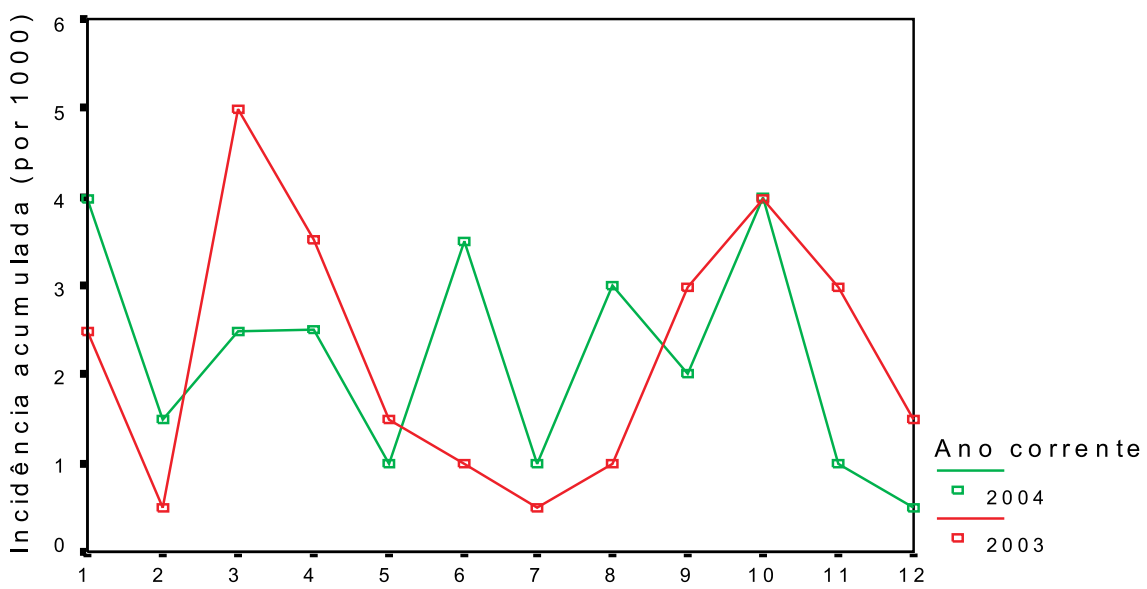

Fonte: Ambulatório de Saúde do Trabalhador, Hospital Universitário de Brasília

Figura 1 Evolução temporal do número de acidentes de trabalho com exposição a material biológico / 1.000 trabalhadores por mês no Hospital Universitário integrante do REPAT nos anos de 2003 e 2004, segundo os meses do ano 
Tabela 5 Distribuição dos acidentes com risco de exposição a material biológico no Hospital Universitário integrante do REPAT, no biênio 2003-2004, segundo os locais de trabalho. Brasília-Brasil

\begin{tabular}{lcccc}
\hline \multicolumn{1}{c}{ Ano } & \multicolumn{2}{c}{2003} & \multicolumn{2}{c}{2004} \\
\multicolumn{1}{c}{ Locais de trabalho } & $n$ & $\%$ & $n$ & $\%$ \\
\hline Clínica médica & 10 & 18,6 & 10 & 19,1 \\
Centro cirúrgico & 10 & 18,6 & 3 & 5,6 \\
Setor de pronto atendimento & 8 & 14,8 & 15 & 28,5 \\
Clínica odontológica & 7 & 13 & 4 & 7,5 \\
Centro obstétrico/maternidade & 6 & 11,1 & 2 & 3,7 \\
Laboratórios & 4 & 7,4 & 6 & 11,2 \\
Clínica cirúrgica & 4 & 7,4 & 4 & 7,5 \\
Pediatria & 2 & 3,7 & 3 & 5,6 \\
Setor de diálise & 1 & 1,8 & 1 & 1,8 \\
UTI & 1 & 1,8 & 5 & 9,3 \\
Sala de vacinas & 1 & 1,8 & - & - \\
Total & 54 & 100 & 53 & 100 \\
\hline
\end{tabular}

Fonte: Ambulatório de Saúde do Trabalhador, Hospital Universitário de Brasília

Tabela 6 Distribuição dos acidentes com risco de exposição a material biológico no Hospital Universitário integrante do REPAT, no biênio 2003-2004, segundo a ocupação. Brasília-Brasil

\begin{tabular}{lcccc}
\hline \multicolumn{1}{c}{ Ano } & \multicolumn{2}{c}{2003} & \multicolumn{2}{c}{2004} \\
\hline Ocupação & $n$ & $\%$ & $n$ & $\%$ \\
\hline Trabalhadírios & 24 & 44,4 & 21 & 39,7 \\
Trabalhadores de enfermagem & 19 & 35,2 & 20 & 37,72 \\
Trabalhadores do setor de limpeza & 4 & 7,4 & 2 & 3,8 \\
Médicos & 4 & 7,4 & 4 & 7,55 \\
Outros & 1 & 1,8 & 3 & 5,66 \\
Total & 2 & 3,8 & 3 & 5,66 \\
\hline
\end{tabular}

Fonte: Ambulatório de Saúde do Trabalhador, Hospital Universitário de Brasília

Embora os números sejam pequenos e impossibilitem uma análise estatística mais ampla dos dados e a interpretação de diferenças estatisticamente significativas, os achados mostram a realidade do hospital estudado e merecem ser destacados, pois podem ser indicativos dos setores do hospital para a implantação de novas estratégias preventivas à ocorrência de AT. No entanto, estudos complementares serão realizados para esclarecer o significado das diferenças encontradas. As unidades de clínica médica, centro cirúrgico, pronto atendimento e odontologia foram os locais onde ocorreram 65\% dos AT em 2003 e as unidades de pronto atendimento, clínica médica, laboratório de análises clínicas e odontologia foram os locais onde foram registrados 64,5\% dos AT em 2004.

A literatura evidencia que os serviços de urgência e centros cirúrgicos são locais onde frequentemente ocorrem os maiores índices de AT com material biológico devido aos numerosos procedimentos realizados com manuseio de material perfurocortante e ao grande número de pacientes/clientes assistidos (MARZIALE, NISHIMURA \& FERREIRA, 2004). A Tabela 6 mostra a freqüência dos acidentes ocorridos no biênio estudado. 
Nos registros, observa-se que 45 acidentes, no biênio, ocorreram com estagiários de Medicina, Odontologia e Enfermagem, os quais representaram 24 (44,44\%) dos episódios acidentais de 2003 e 21 (39,7\%) das ocorrências de 2004. Embora com incidências menores, constatou-se, no ano de 2003, a ocorrência de acidentes entre cirurgiões-dentista $(1,8 \%)$ e trabalhadores do setor administrativo $(1,8 \%)$ e, em 2004 , entre farmacêuticos $(3,8 \%)$ e trabalhadores de lavanderia (1,8\%). Cabe destacar que trabalhadores de diferentes categorias profissionais foram vítimas de acidentes com exposição a material biológico, os quais também merecem atenção quando do planejamento de estratégias preventivas. Outro dado que merece destaque é o pequeno número de acidentes ocorridos entre trabalhadores do serviço de limpeza (3,8\%), uma população exposta ao risco devido ao manuseio e transporte de lixo e dos recipientes de descarte de material perfurocortante.

Estudo realizado na Faculdade de $\mathrm{Me}$ dicina de Minas Gerais comprovou que os estudantes apresentam alto risco de exposição a sangue, conseqüentemente, risco potencial de exposição ao HIV e baixo nível de conhecimento das medidas universais de biossegurança (TOLEDO JUNIOR, 1999). Assim, sugere-se que o referido tema seja abordado em futuras pesquisas.

Em estudo realizado também no Hospital de Brasília, foi constatado que, entre os médicos vítimas de acidentes do trabalho com exposição a material biológico, a maioria não estava utilizando EPI quando da ocorrência do acidente e apresentavam resistência principalmente ao uso de luvas na realização de punções venosas (REPAT, 2007)

O uso de luvas é recomendado internacionalmente por meio das Precauções Padrão e é considerada uma medida preventiva à exposição a material biológico. Apesar de não impedir a perfuração, elas funcionam como uma barreira mecânica auxiliar para diminuir o risco de contato com patógenos transportados pelo sangue. Estudos evidenciaram que o uso de luvas pode reduzir o volume de sangue injetado por agulhas de sutura em 70\% (MAST, WOOLWINW \& GERBERDING, 1993) e a inoculação de sangue por agulhas para o tratamento intravenoso em $35 \%$ a $50 \%$, considerando que parte deste fluido pode permanecer no bisel da agulha (ROSE, 1994). Assim, o uso de luvas, além de impedir o contato de maior quantidade de sangue e outros fluidos corporais com a pele, é extremamente importante na proteção dos profissionais durante a realização de procedimentos com material perfurocortante, dado que os fatores de risco para aquisição de vírus depois da exposição ocupacional dependem da quantidade e do grau de contato do trabalhador com o sangue inoculado.

No entanto, muitas vezes os trabalhadores de saúde desconhecem essas informações e acreditam que o uso deste EPI não diminui a inoculação de sangue, o que tem sido uma barreira para a adequada utilização de luvas principalmente na administração de medicações por via endovenosa (ZAPPAROLI, MARZIALE \& ROBAZZI, 2006).

Para cálculos dos coeficientes de incidência acumulada de acidentes de trabalho por 100 trabalhadores por ano de exposição, foram considerados, no estudo ora apresentado, apenas os trabalhadores formais do hospital. Os resultados encontrados são descritos na Tabela 7.

Observa-se que, embora, em 2004, o risco estimado para os farmacêuticos e bioquímicos tenha sido de 33,33 acidentes por 100 trabalhadores, esses resultados não são estatisticamente significativos devido ao pequeno número de sujeitos expostos e merecem ser tema de futura investigação. No entanto, para fins preventivos, merecem ser mencionados neste estudo. Sendo assim, observa-se ainda que os trabalhadores de laboratório consistentemente apresentam os maiores coeficientes e, como conseqüencia, a probabilidade de sofrer um sinistro nesta categoria é mais do que duas vezes superior do que nas demais categorias nos anos de 2003 e 2004.

Os resultados obtidos mostram que $77,8 \%$ dos trabalhadores acidentados em 2003 e 83\% em 2004 referiram usar EPI quando da ocorrência dos AT. No entanto, $22,2 \%$ em 2003 e 17\% em 2004 não usavam os equipamentos de segurança.

\section{Ações e estratégias preventivas utilizadas para a prevenção dos $\mathrm{AT}$}

As estratégias usadas pelo hospital para a prevenção de AT, segundo dados informados pelo enfermeiro do trabalho, são: treinamento em serviço, realização de visitas de rotina do enfermeiro do trabalho às unidades de trabalho, orientações individuais realizadas na consulta de enfermagem por ocasião dos exames periódicos de rotina. Não existe um pro- 
Tabela 7 Distribuição dos coeficientes de incidência acumulada de acidentes de trabalho por 100 trabalhadores por ano de exposição, com vínculo empregatício, no Hospital Universitário integrante da REPAT, no biênio 2003-2004, segundo a ocupação. Brasília-Brasil

\begin{tabular}{|c|c|c|c|c|}
\hline Categorias & $\begin{array}{c}\text { Número de } \\
\text { trabalhadores expostos } \\
2003\end{array}$ & $\begin{array}{c}2003 \\
\text { Coeficiente de ocorrência } \\
\text { de AT }\end{array}$ & $\begin{array}{c}\text { Número de } \\
\text { trabalhadores expostos } \\
2004\end{array}$ & $\begin{array}{c}2004 \\
\text { Coeficiente de ocorrência } \\
\text { de AT }\end{array}$ \\
\hline Trabalhadores de laboratório & 30 & 13,33 & 29 & 10,34 \\
\hline Trabalhadores de enfermagem & 500 & 9,11 & 525 & 7 \\
\hline Cirurgiões-dentista & 13 & 7,69 & 09 & 0 \\
\hline Trabalhadores do setor de limpeza & 100 & 4 & 112 & 1,78 \\
\hline Trabalhadores do setor administrativo & 30 & 3,33 & 26 & 0 \\
\hline Médico & 199 & 0,5 & 201 & 1,49 \\
\hline Farmacêutico/bioquímico & 09 & 0 & 06 & 33,33 \\
\hline Trabalhadores do setor de lavanderia & 48 & - & 42 & 2,38 \\
\hline
\end{tabular}

Fonte: Ambulatório de Saúde do Trabalhador, Hospital Universitário de Brasília

grama preventivo direcionado a cada um dos setores do hospital considerando as peculiaridades das atividades realizadas, das características dos trabalhadores e das condições do ambiente de trabalho.

\section{Contribuições da REPAT}

Os resultados deste estudo, obtidos por meio de análise do banco de dados da REPAT, apontam para a importância da participação do Hospital Universitário da

\section{Considerações finais}

Considera-se que as estratégias preventivas usadas (treinamento, visitas de inspeção aos locais de trabalho e orientações individuais) devam ser reformuladas e embasadas em métodos educativos e de promoção à saúde ocupacional centrados não só na prevenção de acidentes, mas na melhoria da qualidade de vida no trabalho. Para isso, há necessidade de atenção às formas de organização do trabalho e às condições de trabalho oferecidas pelo hospital a seus trabalhadores.

Embora os dados obtidos impossibilitassem a interpretação de diferenças estatisticamente significativas, os resul-
UnB nesse projeto. Trata-se de informações que poderão orientar futuras ações de prevenção de acidentes do trabalho.

O intercâmbio de informações entre enfermeiro do trabalho e pesquisadores da REPAT possibilitou a elaboração desta pesquisa e, à equipe do SESMT, reavaliar a forma de registro dos AT e as ações empregadas no hospital para prevenção e controle dos AT com material biológico.

tados encontrados mostraram a realidade do hospital e indicaram a necessidade de implantação de novas estratégias preventivas à ocorrência de AT e a necessidade da realização de estudos complementares visando esclarecer o significado das diferenças encontradas.

Considerando-se, ainda, o elevado número de AT sofrido por estagiários, sugere-se que o tema seja objeto de futuras pesquisas, bem como a inserção do conteúdo de segurança no trabalho nos currículos de graduação dos cursos da área da saúde, capacitando os alunos a identifica- 
rem riscos ocupacionais e principalmente como preveni-los.

De acordo com o contrato de trabalho, os acidentes são registrados no hospital em diferentes formulários e muitas vezes de forma incompleta, o que pode dificultar o planejamento de estratégias preventivas mais elaboradas. Assim, o formulário da REPAT pode ser adotado pelo hospital por possibilitar a reunião de todas as informações em um único documento, o qual

\section{Referências}

BOSI, A. R. Proposta de melhoria para redução de acidentes de trabalho no Hospital Universitário de Brasília. 2002. Disponível em: http://repat.eerp.usp. br/publicacao/index.php. Acesso em: 03 ago. 2006.

BRASIL. Ministério da Saúde. Normas éticas em pesquisas envolvendo seres humanos. Diário Oficial da União, n. 183, seção 1, p. 21118, set. 1997.

- Ministério do Trabalho e Emprego. Norma Regulamentadora NR-4. Serviços especializados em engenharia de segurança e medicina do trabalho. Disponível em: http://www.mte.gov.br/ Empregador/ segsau/Legislacao/Normas/ conteudo/nr04/. Acesso em: 10 ago. 2005.

CDC. Centers for Disease Control and Prevention. Guideline for infection control in health care personel. Infect. Control. Hosp. Epidemiol., v. 19, n. 6, p. 455, 2001.

Division of healthcare quality promotion. Surveillance of healthcare personnel with HIV/AIDS [serial online] [cited 2005 Set 26]. Available from: http://www.cdc.gov/ncidod/hip/Blood/ hivpersonnel.htm. Access: 21 nov. 2005.

GODFRE, K. Sharp practice. Nursing Times, v. 97, n. 2, p. 22-24, 2001.

GOMES, G. P. Prevalência de acidentes de trabalho: o caso do hospital universitário de Brasília. Publicações REPAT - Rede de Prevenção de Acidentes de Trabalho. Disponível em: http://repat.eerp.usp. br/publicacao/index.php. Acesso em: 22 jun. 2005.

JAGGER, J. B.; PERRY, J. Exposure safety. Risk phlebotomy with a syringue. Nursing, v. 31, n. 2, p. 73, 2001.

MARZIALE, M. H. P. Subnotificação de acidentes de trabalho com pérfuro- seria complementar à CAT, documento legalmente exigido.

Diante da realidade identificada, a REPAT propõe a implantação de estratégias preventivas a serem efetuadas por meio da educação permanente, visando à identificação dos riscos ocupacionais relacionados à maneira como o trabalho é organizado e executado em cada setor de trabalho, com implantação inicial nas unidades de clínica médica e pronto-socorro.

cortante entre trabalhadores de enfermagem brasileiros. Rev. Bras. Enfermagem, Ribeirão Preto, v. 56, n. 2, p. 164-8, 2003.

MARZIALE, M. H. P.; NISHIMURA, K. Y. N., FERREIRA, M. M. Riscos de contaminação ocasionados por acidentes de trabalho com material pérfuro-cortante entre trabalhadores de enfermagem. Rev. Latino-am Enfermagem, Ribeirão Preto, v. 12, n. 1, p. 36-42, 2004.

MAST, S. T.; WOOLWINW, J. D.; GERBERDING, J. L. Efficacy of gloves in reducing blood volumes transferred during simulated needlestick injury. J. Infec. Dis. v. 168, n. 6, p. 1589-1592,1993.

MEDEIROS, M. A pesquisa científica e a internet. In: ENCONTRO NACIONAL DE CIÊNCIAS DA INFORMAÇÃO, 4, 2003, Salvador. Anais... Salvador: UFBA, 2003. Disponível em: http://www.cinform. ufba.br/iv_anais/artigos/TEXTO14.HTM. 2003. Acesso em: 20 jun. 2005.

NAPOLEÃO, A. A.; ROBAZZI, M. L. do C. C.; MARZIALE, M. H. P. Causas de subnotificação de acidentes do trabalho entre trabalhadores de enfermagem. Rev. Latino-am. Enfermagem, Ribeirão Preto, v. 8, n. 3, p. 119-20, 2000.

NISHIDE, V. M.; BENATTI, M. C. C.; ALEXANDRE, N. M. C. Ocorrência de acidente de trabalho em uma Unidade de Terapia Intensiva. Rev. Latino-am. Enfermagem, Ribeirão Preto, v. 12, n. 2, p. 204-211, 2004.

OSHA. Occupational Safety \& Health Administration. Bloodborne Pathogens. Available from: http: www.CDC.gov/niosh/ elcosh/docs. Access: 2001.

PEREIRA, A. C. M. Acidentes de trabalho com material pérfuro-cortante em profissionais da equipe de enfermagem 
da rede hospitalar pública de Rio BrancoAC. 2004. Trabalho de Conclusão de Curso (Graduação em Enfermagem), Universidade Federal do Acre, Rio Branco, 2004. Disponível em: http://repat. eerp.usp.br/publicacao/index.php. Acesso em: 22 jul. 2005.

PERINE, L. A. A Internet como plataforma em contínua expansão para a pesquisa global. Questões globais. Rev. Eletrônica do Departamento de Estado dos EUA [seriado na Internet]. [citado em: 22 março 2004]; v. 5, n. 3, p. 1, 2000. Disponível em: http://usinfo.state.gov/journals/itgic/1100/ ijgp/ijgp1105.htm. Acesso em: $11 \mathrm{fev}$. 2007.

REPAT. Rede de Prevenção de Acidente de Trabalho. Universidade de São Paulo. Escola de Enfermagem de Ribeirão Preto. Disponível em: http://www.eerp.usp. br/repat. Acesso em: 11 fev. 2007.

ROSE, D. A. Usage patterns and perforation rates for 6.396 gloves from intra-operative procedures at San
Francisco General Hospital /Abstract/ Infec. Control Hosp. Epidemiol., n. 15, p. 349, 1994.

SÃO PAULO (ESTADO). Secretaria do Estado da Saúde. Programa DST/AIDSSP. Vigilância de acidentes com material biológico. Bol. Epidemiol. SINABIO, p. 20, 2002.

TOLEDO JÚNIOR, A. C. C. T.

Conhecimento, atitudes e comportamentos frente ao risco ocupacional de exposição ao HIV entre estudantes de Medicina da Faculdade de Medicina da Universidade Federal de Minas Gerais. Rev. Soc. Bras. Med. Trop. [seriado on line], Uberaba, v. 32, n. 5, p. 509-15, 1999. Disponível em: http://repat.eerp.usp.br/publicacao/index. php. Acesso em: 22 jun. 2006.

ZAPPAROLI, A. S.; MARZIALE, M. H. P.; ROBAZZI, M. L. C. C. Práctica segura del uso de guantes en la puncion venosa por los trabajadores de enfermeria. Ciencia y enfermeria, v. 12, n. 2, p. 63-72, 2006. 